\title{
ON THE CONFIGURATIONS OF CENTERS OF PLANAR HAMILTONIAN KOLMOGOROV CUBIC POLYNOMIAL DIFFERENTIAL SYSTEMS
}

\author{
JAUME LLIBRE ${ }^{1}$ AND DONGMEI XIAO ${ }^{2 \dagger}$
}

\begin{abstract}
We study the kind of centers that Hamiltonian Kolmogorov cubic polynomial differential systems can exhibit. Moreover, we analyze the possible configurations of these centers with respect to the invariant coordinates axes, and obtain that the real algebraic curve $x y\left(a+b x+c y+d x^{2}+e x y+f y^{2}\right)=h$ has at most four families of level ovals in $\mathbb{R}^{2}$ for all real parameters $a, b, c, d, e, f$ and $h$.
\end{abstract}

\section{INTRODUCTION AND STATEMENT OF THE MAIN RESUltS}

An equilibrium $p$ of a differential system in $\mathbb{R}^{2}$ is a center if there is a neighborhood $U$ of $p$ such that $p$ is a unique equilibrium in $U$ and $U \backslash\{p\}$ is filled by the periodic orbits. The equilibrium $p$ is a focus if there is a neighborhood $U$ of $p$ such that all the orbits in $U \backslash\{p\}$ spiral tending to $p$ either in forward, or in backward time. These notions of center and focus go back to Poincaré [22] and Dulac [9].

In the qualitative theory of planar polynomial differential systems, the problem of distinguish between a focus or a center (known simply as the center-focus problem), and the problem to know the possible configurations of centers are two very important topics, which are related to the Hilbert 16th problem, see Hilbert [12], Ilyashenko [14], Li [18].

For the quadratic polynomial differential systems the center-focus problem and the possible configurations of their centers were solved by Bautin [3], Kapteyn [15, 16], Schlomiuk [23], Vulpe [26], Żołądek [29]. However, the two problems are unsolved for cubic polynomial differential systems. There are many works on the centers for some different subclasses of cubic differential systems. For example, the centers of the cubic polynomial differential systems without quadratic terms have been determined by Malkin [21], Vulpe and Sibirskii [27], Żołạdek [30] and references therein. The classification of reversible cubic polynomial differential systems with a center has been done by Żołądek [31,32], and Buzzi et al [4]. The Hamiltonian linear type centers and the nilpotent ones of cubic polynomial differential systems without quadratic terms have been classified by Colak et al [5-8].

2010 Mathematics Subject Classification. 37K10, 37C27, 37K05.

Key words and phrases. Hamiltonian system, Kolmogorov systems, cubic polynomial differential systems, centers, configuration of centers.

$\dagger$ Corresponding author. 
In this paper we study the centers and their configurations for the Hamiltonian cubic polynomial differential systems having two invariant straight lines which intersect. Since with an affine transformation these two invariant straight lines go the axes of coordinates, these systems become Kolmogorov systems [17] which allows to describe dynamics of species population $[13,24]$. So it is a very interesting topic to study their dynamics.

Hamiltonian systems come originally from the physics, but also play an important role in the dynamical system theory [2]. Hamiltonian systems in the plane are the easiest differential systems having a first integral. The Kolmogorov polynomial differential systems [17] in the plane generalize the Lotka-Volterra polynomial differential systems $[20,25]$ of degree 2 to higher degree. In this paper we pay our attention on such subclass of cubic polynomial differential systems which are Hamiltonian and Kolmogorov systems, and we shall read them as a HamiltonianKolmogorov system. form

Let $(x, y)$ be the coordinates of $\mathbb{R}^{2}$. We consider Kolmogorov systems of the

$$
\begin{aligned}
& \dot{x}=x P_{1}(x, y), \\
& \dot{y}=y P_{2}(x, y)
\end{aligned}
$$

in $\mathbb{R}^{2}$, where the dot in all this paper means derivative with respect to the independent variable $t$, and the $P_{i}(x, y)$ for $i=1,2$ are polynomials in the variables $x$ and $y$ such that the maximum of their degrees is two. So system (1) is a cubic polynomial differential system.

In the next result we characterize the Kolmogorov systems (1) which are Hamiltonian systems.

Theorem 1. Assume that the maximum degree of the polynomials $P_{i}(x, y)$ is two for $i=1,2$. Then system (1) is a Hamiltonian system if and only if its Hamiltonian is of the form

$$
H(x, y)=x y\left(a+b x+c y+d x^{2}+e x y+f y^{2}\right)
$$

where $a, b, c, d, e$ and $f$ are real parameters.

Theorem 1 is proved in section 2. Therefore the Hamiltonian-Kolmogorov polynomial systems of degree three are

$$
\begin{aligned}
& \dot{x}=-x\left(a+b x+2 c y+d x^{2}+2 e x y+3 f y^{2}\right), \\
& \dot{y}=y\left(a+2 b x+c y+3 d x^{2}+2 e x y+f y^{2}\right),
\end{aligned}
$$

where $a, b, c, d, e$ and $f$ are real parameters. For brevity we denote this system as $H K$-systems in what follows.

A polynomial differential system in the plane having a center at the origin of coordinates, after a linear change of variables and a rescaling of the time variable (if necessary), can be written in one of the following three forms:

$$
\begin{aligned}
& \dot{x}=-y+X_{2}(x, y), \\
& \dot{y}=x+Y_{2}(x, y),
\end{aligned}
$$


called a linear type center,

$$
\begin{aligned}
\dot{x} & =y+X_{2}(x, y), \\
\dot{y} & =Y_{2}(x, y),
\end{aligned}
$$

called a nilpotent center,

$$
\begin{aligned}
& \dot{x}=X_{2}(x, y), \\
& \dot{y}=Y_{2}(x, y),
\end{aligned}
$$

called a degenerate center, where $X_{2}(x, y)$ and $Y_{2}(x, y)$ are polynomials starting at least with terms of second order. For additional information about these three classes of centers see for instance [19], and the references therein.

The objective of this paper is to study the centers and their configurations of the HK-systems (3). In other words we study the existence and configurations of level ovals for planar real algebraic curve $H(x, y)=h, h \in \mathbb{R}$.

Our main result is the following.

Theorem 2. Consider the HK-systems (3) defined by the Hamiltonian (2). Then a system (3) has at most four centers and the following statements hold.

(i) If HK-system (3) has non-isolated equilibria, i.e. there is an infinite number of equilibria, then this system has at most one center, which is linear type. Hence, there exist the values of $a, b, c, d, e$ and $f$ such that $H(x, y)=h$ has no level ovals for all real $h$, and there exist other values of $a, b, c, d, e$ and $f$ such that $H(x, y)=h$ has only one family of level ovals for all real $h$.

(ii) HK-system (3) has neither degenerate centers nor two nilpotent centers for any real $a, b, c, d, e$ and $f$.

(iii) If HK-system (3) has a unique center, then this center can be of linear type or nilpotent. That is, there is only a family of level ovals of $H(x, y)=h$ for all real h, see Figure 1.

(iv) If HK-system (3) has two centers, then the two centers are either two linear type centers, or one linear type center and one nilpotent center. And there are $H K$-systems with two centers realizing all possible configurations with respect to the coordinates axes; i.e. two centers in the same quadrant (see Figure 2), two centers in different opposite quadrants (see Figure 3), and two centers in different consecutive quadrants (see Figure 4). Hence, the level ovals of $H(x, y)=h$ have two families for all real $h$.

(v) If HK-system (3) has three centers, then they are linear type and there are HK-systems with three centers realizing all possible configurations with respect to the coordinates axes; i.e. three centers in the same quadrant (see Figure 5), two centers in one quadrant and the other in a consecutive quadrant (see Figure 6), two centers in one quadrant and the other in the opposite quadrant (see Figure 7), and three centers in three different quadrants (see Figure 8). Moreover, the level ovals of $H(x, y)=h$ have at least three families and at most four families for all real $h$.

(vi) If HK-system (3) has four centers, then they are linear type. The level ovals of the corresponding Hamiltonian function, $H(x, y)=h$, have exactly four families for all real number $h$. Moreover, there are HK-systems with four centers realizing two kinds of configurations with respect to the coordinates 
axes. One configuration is four centers in the four different quadrants (see Figure 9); and the other is two centers in the same quadrant, and each one of the other two centers is located in a different adjacent quadrant (see Figure 10).

Theorem 2 will be proved in section 3 . As a direct application of Theorem 2, we have

Corollary 3. The real algebraic curve

$$
x y\left(a+b x+c y+d x^{2}+e x y+f y^{2}\right)=h
$$

has at most four families of level ovals in $\mathbb{R}^{2}$ for all real parameters $a, b, c, d, e, f$ and $h$.

\section{Preliminaries}

In this section we first give the proof of Theorem 1 , then discuss the equilibria of the systems (3) and recall some definitions of equilibrium and related results.

Proof of Theorem 1. Consider an arbitrary polynomial in the variables $x$ and $y$ of degree 4

$$
H=\sum_{i+j=1}^{4} a_{i j} x^{i} y^{j} .
$$

We shall force that $H$ defines a Hamiltonian system

$$
\dot{x}=-H_{y}, \quad \dot{y}=H_{x} .
$$

with form of the system (1). Then $H_{y}$ appears $x$ as a common factor and $y$ appears as a common factor in $H_{x}$. In order that we must take $a_{01}=a_{02}=a_{03}=a_{04}=0$, and $a_{10}=a_{20}=a_{30}=a_{40}=0$. Therefore the Hamiltonian system (7) becomes a Kolmogorov system (1) if and only if

$$
H=x y\left(a_{11}+a_{21} x+a_{12} y+a_{31} x^{2}+a_{22} x y+a_{13} y^{2}\right) .
$$

Renaming the coefficients of $H$ the theorem follows.

We now discuss the equilibria of system (3) in $\mathbb{R}^{2}$. It is clear that the number of equilibrium of system (3) is finite in $\mathbb{R}^{2}$ if and only if this polynomial vector field

$\left(p_{1}(x, y), p_{2}(x, y)\right)=\left(-x\left(a+b x+2 c y+d x^{2}+2 e x y+3 f y^{2}\right), y\left(a+2 b x+c y+3 d x^{2}+2 e x y+f y^{2}\right)\right)$

does not have a common non-constant component by Bézout's theorem (see for instance [11]). Hence, if the two polynomials $p_{1}(x, y)$ and $p_{2}(x, y)$ have a non-constant common component (or called common divisor), then system (3) has non-isolated equilibria in $\mathbb{R}^{2}$. The following lemma gives the necessary and sufficient conditions for the existence of a non-constant common divisor of $p_{1}(x, y)$ and $p_{2}(x, y)$.

Lemma 4. Polynomials $p_{1}(x, y)$ and $p_{2}(x, y)$ have a non-constant common divisor if and only if one of the following conditions in Table 1 holds, where conditions C 9 and $C 12$ are equivalent. 


\begin{tabular}{|c|c|c|}
\hline List & Conditions & non-constant common divisors \\
\hline$C 1$ & $a=b=c=e=f=0, d \neq 0$ & $x^{2}$ \\
\hline$C 2$ & $a=b=c=d=e=0, f \neq 0$ & $y^{2}$ \\
\hline$C 3$ & $a=b=c=d=f=0, e \neq 0$ & $x y$ \\
\hline$C 4$ & $b=c=d=f=0, a e \neq 0$ & $a+2 e x y$ \\
\hline$C 5$ & $a=c=f=0, b \neq 0($ or $d e \neq 0)$ & $x$ \\
\hline$C 6$ & $a=b=d=0, c \neq 0($ or $e f \neq 0)$ & $y$ \\
\hline$C 7$ & $c=e=f=0, b d \neq 0, a=\frac{b^{2}}{4 d}$ & $x+\frac{b}{2 d}$ \\
\hline$C 8$ & $a=b=c=0, e f \neq 0, d=\frac{e^{2}}{4 f}$ & $x+\frac{2 f}{e} y$ \\
\hline$C 9$ & $b e f \neq 0, a=\frac{b^{2} f}{e^{2}}, c=\frac{2 b f}{e}, d=\frac{e^{2}}{4 f}$ & $x+\frac{2 f}{e} y+\frac{2 b f}{e^{2}}$ \\
\hline$C 10$ & $b=d=e=0, a c \neq 0, f=\frac{c^{2}}{4 a}$ & $y+\frac{2 a}{c}$ \\
\hline$C 11$ & $a=b=c=0, d e \neq 0, f=\frac{e^{2}}{4 d}$ & $y+\frac{2 d}{e} x$ \\
\hline$C 12$ & $a c e \neq 0, b=\frac{2 a e}{c}, d=\frac{a e^{2}}{c^{2}}, f=\frac{c^{2}}{4 a}$ & $y+\frac{2 a e}{c^{2}} x+\frac{2 a}{c}$ \\
\hline
\end{tabular}

Table 1: The necessary and sufficient conditions for non-constant common divisor

Proof. It is clear that polynomials $p_{1}(x, y)$ and $p_{2}(x, y)$ do not have a common divisor which is a polynomial of $(x, y)$ with degree three. Hence, we only look for the common divisor which is a polynomial of $(x, y)$ with degree two or one.

By straightforward calculation, we obtain that polynomials $p_{1}(x, y)$ and $p_{2}(x, y)$ have a common divisor with degree two if and only if the common divisor has one of the four forms $x^{2}, y^{2}, x y$ and $a+2 e x y$ for any real parameters $a, b, c, d, e$ and $f$. Hence, the conditions $C 1-C 4$ for the corresponding common divisor with degree two can be given in the first four rows in Table 1.

We now look for the common divisor of polynomials $p_{1}(x, y)$ and $p_{2}(x, y)$ with degree only one. Note that

$$
\begin{aligned}
& p_{1}(x, y)=-x\left(a+b x+2 c y+d x^{2}+2 e x y+3 f y^{2}\right), \\
& p_{2}(x, y)=y\left(a+2 b x+c y+3 d x^{2}+2 e x y+f y^{2}\right) .
\end{aligned}
$$

Suppose that $y-s-t x$ is a common divisor of $a+b x+2 c y+d x^{2}+2 e x y+3 f y^{2}$ and $a+2 b x+c y+3 d x^{2}+2 e x y+f y^{2}$, where $s$ and $t$ are undetermined real parameters. Then for any real $x$,

$$
\begin{aligned}
& a+b x+2 c(s+t x)+d x^{2}+2 e x(s+t x)+3 f(s+t x)^{2} \equiv 0 \\
& a+2 b x+c(s+t x)+3 d x^{2}+2 e x(s+t x)+f(s+t x)^{2} \equiv 0 .
\end{aligned}
$$

Thus,

$$
\begin{aligned}
& a+2 c s+3 f s^{2}=0, \\
& a+c s+f s^{2}=0, \\
& b+2 c t+2 e s+6 f s t=0, \\
& 2 b+c t+2 e s+2 f s t=0, \\
& d+2 e t+3 f t^{2}=0, \\
& 3 d+2 e t+f t^{2}=0 .
\end{aligned}
$$


We divide four cases of $(s, t)$ to study the solutions of system (9).

Case 1: $s=t=0$. From (9) it follows that $a=b=d=0$. Hence, $a+b x+2 c y+$ $d x^{2}+2 e x y+3 f y^{2}$ and $a+2 b x+c y+3 d x^{2}+2 e x y+f y^{2}$ has a common divisor $y$ if $a=b=d=0$ and $c^{2}+e^{2}+f^{2} \neq 0$. However, if we require this common divisor to be the only polynomial with degree one, then $a=b=d=0$ and $c \neq 0$, or $a=b=d=0$ and ef $\neq 0$, which is the condition $C 6$ in Table 1 .

Case 2: $s \neq 0$ and $t=0$. The equation (9) becomes

$$
\begin{aligned}
& a+2 c s+3 f s^{2}=0 \\
& a+c s+f s^{2}=0 \\
& b=d=e=0 .
\end{aligned}
$$

Therefore, when $b=d=e=0, a c \neq 0$ and $f=\frac{c^{2}}{4 a}$, we have $y+\frac{2 a}{c}$ is the common divisor of $a+b x+2 c y+d x^{2}+2 e x y+3 f y^{2}$ and $a+2 b x+c y+3 d x^{2}+2 e x y+f y^{2}$. In fact, for this moment we have

$$
a+b x+2 c y+d x^{2}+2 e x y+3 f y^{2}=\frac{c^{2}}{4 a}\left(y+\frac{2 a}{c}\right)\left(3 y+\frac{2 a}{c}\right),
$$

and

$$
a+2 b x+c y+3 d x^{2}+2 e x y+f y^{2}=\frac{c^{2}}{4 a}\left(y+\frac{2 a}{c}\right)^{2} .
$$

Case 3: $s=0$ and $t \neq 0$. The equation (9) becomes

$$
\begin{aligned}
& a=b=c=0 \\
& d+2 e t+3 f t^{2}=0 \\
& 3 d+2 e t+f t^{2}=0
\end{aligned}
$$

Therefore, when $a=b=c=0, d e \neq 0$ and $f=\frac{e^{2}}{4 d}$, we have $y+\frac{2 d}{e} x$ is the common divisor of $a+b x+2 c y+d x^{2}+2 e x y+3 f y^{2}$ and $a+2 b x+c y+3 d x^{2}+2 e x y+f y^{2}$. In fact, for this moment we have

$$
a+b x+2 c y+d x^{2}+2 e x y+3 f y^{2}=\frac{3 e^{2}}{4 d}\left(y+\frac{2 d}{e} x\right)\left(y+\frac{2 d}{3 e} x\right),
$$

and

$$
a+2 b x+c y+3 d x^{2}+2 e x y+f y^{2}=\frac{3 e^{2}}{4 d}\left(y+\frac{2 d}{e} x\right)\left(y+\frac{6 d}{e} x\right) .
$$

Case 4: $s \neq 0$ and $t \neq 0$. From the first two equations in (9) it follows that

$$
s=-\frac{2 a}{c} \text { with } a c \neq 0 \text {. }
$$

From the last two equations in (9) it follows that

$$
t=-\frac{2 d}{e} \text { with } d e \neq 0
$$

Plug (10) and (11) into (9), we obtain

$$
b=\frac{2 a e}{c}, d=\frac{a e^{2}}{c^{2}}, f=\frac{c^{2}}{4 a} .
$$

Hence when $a c e \neq 0, b=\frac{2 a e}{c}, d=\frac{a e^{2}}{c^{2}}$ and $f=\frac{c^{2}}{4 a}, y+\frac{2 a e}{c^{2}} x+\frac{2 a}{c}$ is a common divisor of $a+b x+2 c y+d x^{2}+2 e x y+3 f y^{2}$ and $a+2 b x+c y+3 d x^{2}+2 e x y+f y^{2}$. 
In fact, for this moment we have

$$
a+b x+2 c y+d x^{2}+2 e x y+3 f y^{2}=\frac{c^{2}}{4 a}\left(y+\frac{2 a e}{c^{2}} x+\frac{2 a}{c}\right)\left(3 y+\frac{2 a e}{c^{2}} x+\frac{2 a}{c}\right),
$$

and

$$
a+2 b x+c y+3 d x^{2}+2 e x y+f y^{2}=\frac{c^{2}}{4 a}\left(y+\frac{2 a e}{c^{2}} x+\frac{2 a}{c}\right)\left(y+\frac{6 a e}{c^{2}} x+\frac{2 a}{c}\right) .
$$

Summarizing the above analysis, we obtain the necessary and sufficient conditions that $p_{1}(x, y)$ and $p_{2}(x, y)$ have common divisors $y, y-s, y-t x$ and $y-s-t x$, respectively. This leads to the conditions $C 6$ and $C 10-C 12$ in Table 1.

Using the similar arguments, one can discuss the common divisor of $a+b x+$ $2 c y+d x^{2}+2 e x y+3 f y^{2}$ and $a+2 b x+c y+3 d x^{2}+2 e x y+f y^{2}$ with form $x-s-t y$. Hence, we obtain the conditions shown in the condition $C 5$ and $C 7-C 9$ in Table 1. This proof is complete.

Remark 5. The eleven conditions from $C 1$ to $C 11$ in Table 1 give the necessary and sufficient conditions that the polynomial $H(x, y)$ can be factorized in real number field. This gives all possible real branch curves of real algebraic curve $H(x, y)=0$.

From Bézout's theorem and the straightforward calculation, we have

Theorem 6. The following statements hold on the equilibria of HK-system (3).

(I) If polynomials $p_{1}(x, y)$ and $p_{2}(x, y)$ have a non-constant common divisor $g(x, y)$, then HK-system (3) has non-isolated equilibria, and each point on the curve $g(x, y)=0$ is an equilibrium of the HK-system (3). Hence HKsystem (3) has infinitely many equilibria.

(II) If polynomials $p_{1}(x, y)$ and $p_{2}(x, y)$ do not have non-constant common divisors, then HK-system (3) has at most nine finite equilibria with five of them located at the coordinates axes and the other four are in the interior of the quadrants in $\mathbb{R}^{2}$.

In two dimension phase plane $\mathbb{R}^{2}$, we say that an equilibrium is non-elementary if both of the eigenvalues of the linear part of the vector field at that point are zero, and elementary otherwise. A non-elementary equilibrium is called degenerate if the linear part is identically zero, otherwise it is called nilpotent. An elementary equilibrium is hyperbolic if the both eigenvalues of the linear part at the equilibrium have non-zero real part, and it is called semi-hyperbolic if one and only one of the two eigenvalues is zero. The unique elementary equilibrium which is neither hyperbolic nor semi-hyperbolic is the one with purely imaginary eigenvalues, which only can be focus or center, see for more details $[10,28]$.

The local phase portraits at the nilpotent equilibrium were classified by Andreev [1], see also Theorem 3.5 of [10]. We summarize the conditions which characterize that a nilpotent equilibrium is either a focus or a center as follows.

Lemma 7. Let $(0,0)$ be an isolated equilibrium point of the analytic differential system

$$
\dot{x}=y+A(x, y), \quad \dot{y}=B(x, y),
$$


defined in a neighborhood of the point $(0,0)$, and assume that $A(x, y)$ and $B(x, y)$ start at least with terms of degree two in $x$ and $y$. Let $y=f(x)$ be the solution of the equation $y+A(x, y)=0$ in a neighborhood of the point $(0,0)$, and consider $F(x)=B(x, f(x))$ and $G(x)=(\partial A / \partial x+\partial B / \partial y)(x, f(x))$. Then the origin of system (12) is a center or a focus if and only if one of the following conditions holds.

(i) $G(x) \equiv 0, F(x)=a x^{m}+o\left(x^{m}\right), m>1$ is an odd integer and $a<0$.

(ii) $G(x)=b x^{n}+o\left(x^{n}\right), F(x)=a x^{m}+o\left(x^{m}\right), m>1$ is an odd integer, $a<0$, and either $m<2 n+1$ or both $m=2 n+1$ and $b^{2}+4 a(n+1)<0$.

For the convenience to apply Lemma 7 we recall some characteristics of a nilpotent equilibrium. As it is known that a planar analytic differential system

$$
\dot{u}=f(u, v), \quad \dot{v}=g(u, v),
$$

has a nilpotent equilibrium at $\left(u_{0}, v_{0}\right)$, then the Jacobian matrix $M$ of system (13) at $\left(u_{0}, v_{0}\right)$ must be

$$
M=\left(\begin{array}{cc}
\frac{\partial f\left(u_{0}, v_{0}\right)}{\partial u} & \frac{\partial f\left(u_{0}, v_{0}\right)}{\partial v} \\
\frac{\partial g\left(u_{0}, v_{0}\right)}{\partial u} & \frac{\partial g\left(u_{0}, v_{0}\right)}{\partial v}
\end{array}\right) \triangleq\left(\begin{array}{cc}
m_{1} & m_{2} \\
m_{3} & -m_{1}
\end{array}\right),
$$

where $m_{1}^{2}+m_{2} m_{3}=0$, and $m_{1}^{2}+m_{2}^{2}+m_{3}^{2} \neq 0$.

By an affine transformation

$$
\left(\begin{array}{l}
x \\
y
\end{array}\right)=P\left(\begin{array}{l}
u-u_{0} \\
v-v_{0}
\end{array}\right)
$$

system (13) can be transformed into system (12), where matrix $P$ depends on $m_{1}$, $m_{2}$ and $m_{3}$, which has one of the following three forms.

(i) If $m_{1} \neq 0$, then $m_{2} \neq 0$ and $m_{3} \neq 0$. Hence, $P=\left(\begin{array}{cc}0 & -\frac{m_{2}}{m_{1}^{2}} \\ 1 & \frac{m_{2}}{m_{1}}\end{array}\right)$;

(ii) If $m_{1}=0$ and $m_{2} \neq 0$, then $m_{3}=0$. Hence, $P=\left(\begin{array}{cc}\frac{1}{m_{2}} & 0 \\ 0 & 1\end{array}\right)$;

(iii) If $m_{1}=0$ and $m_{3} \neq 0$, then $m_{2}=0$. Hence, $P=\left(\begin{array}{cc}0 & -\frac{1}{m_{3}} \\ -1 & 0\end{array}\right)$.

Consider a Hamiltonian system with a nilpotent equilibrium, we have the following theorem.

Theorem 8. Suppose that system (13) is a Hamiltonian system with a nilpotent equilibrium at $\left(u_{0}, v_{0}\right)$, then this nilpotent equilibrium is a center if and only if the case (i) in lemma 7 holds, that is, $G(x) \equiv 0$ and $F(x)=a x^{m}+o\left(x^{m}\right)$, where $m>1$ is an odd integer and $a<0$.

Proof. Since system (13) is a Hamiltonian system, there exists an analytic function $H(u, v)$ such that

$$
\frac{\partial H(u, v)}{\partial v} \triangleq H_{v}(u, v)=f(u, v), \frac{\partial H(u, v)}{\partial u} \triangleq H_{u}(u, v)=-g(u, v) .
$$


Thus, system (13) can be written as

$$
\dot{u}=H_{v}(u, v), \quad \dot{v}=-H_{u}(u, v)
$$

with $H_{v}\left(u_{0}, v_{0}\right)=-H_{u}\left(u_{0}, v_{0}\right)=0$.

Using the affine transformation (14) and its inverse transformation

$$
\left(\begin{array}{l}
u(x, y) \\
v(x, y)
\end{array}\right)=P^{-1}\left(\begin{array}{l}
x \\
y
\end{array}\right)+\left(\begin{array}{l}
u_{0} \\
v_{0}
\end{array}\right),
$$

system (15) can be transformed to

$$
\left(\begin{array}{c}
\dot{x} \\
\dot{y}
\end{array}\right)=P\left(\begin{array}{cc}
0 & 1 \\
-1 & 0
\end{array}\right) P^{T}\left(\begin{array}{c}
\tilde{H}_{x}(x, y) \\
\left.\tilde{H}_{y}(x, y)\right)
\end{array}\right),
$$

where $\tilde{H}(x, y)=H(u(x, y), v(x, y))$ and $P^{T}$ is the transpose of matrix $P$.

Let

$$
Q=P\left(\begin{array}{cc}
0 & 1 \\
-1 & 0
\end{array}\right) P^{T}
$$

Depending on the Jacobian matrix $M$ of system (13) at nilpotent equilibrium $\left(u_{0}, v_{0}\right)$, by direct computation we obtain three forms of $Q$ as follows.

$$
\begin{aligned}
& \text { (i) } Q=\left(\begin{array}{cc}
0 & \frac{m_{2}}{m_{1}^{2}} \\
-\frac{m_{2}}{m_{1}^{2}} & 0
\end{array}\right) \text { if } m_{1} \neq 0 \\
& \text { (ii) } Q=\left(\begin{array}{cc}
0 & \frac{1}{m_{2}} \\
-\frac{1}{m_{2}} & 0
\end{array}\right) \text { if } m_{1}=0 \text { and } m_{2} \neq 0 \text {; } \\
& \text { (iii) } Q=\left(\begin{array}{cc}
0 & -\frac{1}{m_{3}} \\
\frac{1}{m_{3}} & 0
\end{array}\right) \text { if } m_{1}=0 \text { and } m_{3} \neq 0 .
\end{aligned}
$$

This leads that system (17) is a Hamiltonian system even though $P$ is not a symplectic matrix if nonzero $m_{i} \neq 1$ for some $i \in\{1,2,3\}$. Following the notations in Lemma 7, we have

$$
G(x)=c\left(\frac{\partial^{2} \tilde{H}(x, y)}{\partial x \partial y}-\frac{\partial^{2} \tilde{H}(x, y)}{\partial y \partial x}\right) \equiv 0,
$$

where $c$ is one of $\frac{m_{2}}{m_{1}^{2}}, \frac{1}{m_{2}}$ and $-\frac{1}{m_{3}}$.

Note that this system has a first integral defined at the origin. Consequently, the origin is a nilpotent center if and only if the case (i) in lemma 7 holds, that is, $G(x) \equiv 0$ and $F(x)=a x^{m}+o\left(x^{m}\right)$, where $m>1$ is an odd integer and $a<0$ by Lemma 7.

We finish the proof.

\section{Proof of Theorem 2}

We shall prove Theorem 2 statement by statement in this section.

Proof of statement (i) of Theorem 2. Since the $x$-axis and $y$-axis are invariant by the flow of HK-system (3), either the equilibrium on the coordinates axes or the non-isolated equilibrium can not become a center by the definition of center. If 
HK-system (3) does not have a center, then the algebraic curve $H(x, y)=h$ does not have level ovals in $\mathbb{R}^{2}$.

From Theorem 6, HK-system (3) has at most four isolated equilibria outside the coordinates axes. Hence, HK-system (3) has at most four centers in the interior of quadrants of $\mathbb{R}^{2}$.

We now prove the first statement (i) of Theorem 2: if HK-system (3) has nonisolated equilibria, then this system has at most one center, which is linear type.

From Lemma 4, we know that HK-system (3) has non-isolated equilibria if and only if one of conditions listed in Table 1 holds. By straightforward calculation, HKsystem (3) has no isolated equilibrium outside the coordinates axes for conditions $C 1-C 4, C 7, C 8, C 10$ and $C 11$ in Table 1 . Hence, under these conditions HKsystem (3) has no center equilibrium, which implies that $H(x, y)=h$ has no level ovals for all real $h$.

When condition $C 5: a=c=f=0, b \neq 0$ (or $d e \neq 0$ ) holds, HK-system (3) has an isolated equilibrium outside the coordinates axes if and only if $b d e \neq 0$. In the case $a=c=f=0, b d e \neq 0$, HK-system (3) has a unique isolated equilibrium at $\left(-\frac{b}{2 d},-\frac{b}{4 e}\right)$ with eigenvalues $\pm \frac{b^{2}}{2 \sqrt{2}|d|} i$, which is a linear type center. Hence, there is only one family of level ovals for $H(x, y)=x^{2} y(b+d x+e y)=h, h \in \mathbb{R}$.

Similarly, we can discuss the cases $C 6$ and $C 9$ (or $C 12$ ). For clarity, we list them in Table 2. Thus, statement (i) is proved.

\begin{tabular}{|c|c|c|c|}
\hline \multicolumn{2}{|r|}{ Conditions } & Center & Level ovals of $H(x, y)=h$ \\
\hline \multicolumn{2}{|c|}{$\mathrm{C} 1$} & none & $d x^{3} y=h$, none \\
\hline \multicolumn{2}{|r|}{$\mathrm{C} 2$} & none & $f x^{3} y=h$, none \\
\hline \multicolumn{2}{|r|}{ C3 } & none & $e x^{2} y^{2}=h$, none \\
\hline \multicolumn{2}{|r|}{$\mathrm{C} 4$} & none & $x y(a+e x y)=h$, none \\
\hline \multirow{2}{*}{$C 5$} & $b=0, d e \neq 0$ & none & $x^{2} y(d x+e y)=h$, none \\
\hline & $b d e \neq 0$ & $\left(-\frac{b}{2 d},-\frac{b}{4 e}\right)$ & $x^{2} y(b+d x+e y)=h$, a family of ovals \\
\hline \multirow{2}{*}{$C 6$} & $c=0$, ef $\neq 0$ & none & $x y^{2}(e x+f y)=h$, none \\
\hline & $c e f \neq 0$ & $\left(-\frac{c}{4 e},-\frac{c}{2 f}\right)$ & $x y^{2}(c+e x+f y)=h$, a family of ovals \\
\hline \multicolumn{2}{|r|}{$\mathrm{C} 7$} & none & $x y(b+2 d x)^{2} y /(4 d)=h$, none \\
\hline \multicolumn{2}{|r|}{$\mathrm{C} 8$} & none & $x y(e x+2 f y)^{2} /(4 f)=h$, none \\
\hline \multicolumn{2}{|r|}{ C9 } & $\left(-\frac{b f}{2 e^{2}},-\frac{b}{4 e}\right)$ & $x y\left(2 b f+e^{2} x+2 e f y\right)^{2} /\left(4 e^{2} f\right)=h$, a family of ovals \\
\hline \multicolumn{2}{|r|}{$\mathrm{C} 10$} & none & $x y(2 a+c y)^{2} /(4 a)=h$, none \\
\hline \multicolumn{2}{|r|}{ C11 } & none & $x y(2 d x+e y)^{2} /(4 d)=h$, none \\
\hline
\end{tabular}

Table 2: Center of HK-system (3) with non-isolated equilibria

It is clear that HK-system (3) has no center if HK-system (3) does not have any isolated equilibria outside the coordinates axes. In the proof of the remainder statements of Theorem 2, we always assume that HK-system (3) has an isolated equilibrium outside the coordinates axes. Without loss of generality, it is in the positive quadrant, and consequently we can assume that it is $(x, y)=(\alpha, \beta)$ with 
$\alpha$ and $\beta$ positive real numbers. Then, scaling the variables $x$ and $y$ if necessary, we can suppose that $\alpha=\beta=1$. In short, in the rest of the proofs of the statements of Theorem 2 we always assume that the point $(1,1)$ will be an equilibrium of HKsystem (3). Hence, two parameters of $a, b, c, d, e$ and $f$ can be determined by the other four parameters, e.g.

$$
e=-\frac{1}{4}(2 a+5 b+c+8 d), \quad f=\frac{1}{2}(b-c+2 d) .
$$

Therefore HK-system (3) with equilibrium $(1,1)$ becomes

$$
\begin{aligned}
\dot{x}= & -x\left(a+b x+d x^{2}+2 c y-a x y-\frac{5}{2} b x y-\frac{1}{2} c x y-4 d x y\right. \\
& \left.+\frac{3}{2} b y^{2}-\frac{3}{2} c y^{2}+3 d y^{2}\right), \\
\dot{y}= & y\left(a+2 b x+3 d x^{2}+c y-a x y-\frac{5}{2} b x y-\frac{1}{2} c x y\right. \\
& \left.-4 d x y+b y^{2} / 2-c y^{2} / 2+d y^{2}\right) .
\end{aligned}
$$

The Jacobi matrix of HK-system $(18)$ at $(1,1)$ is

$$
M=\left(\begin{array}{cc}
(2 a+3 b+c+4 d) / 2 & (2 a-b+3 c-4 d) / 2 \\
-(2 a+b+c-4 d) / 2 & -(2 a+3 b+c+4 d) / 2
\end{array}\right),
$$

and the determinant of $M$ is denoted by $\Delta$,

$$
\Delta=a c-b c-3 a b-\frac{5 b^{2}-c^{2}}{2}-2(4 a+3 b+3 c) d .
$$

Proof of statements (ii) of Theorem 2. To prove statement (ii) of Theorem 2: HKsystem (3) has neither degenerate centers nor two nilpotent centers for any real $a, b, c, d, e$ and $f$, we first prove that HK-system (3) does not have a degenerate center.

Assume that HK-system (3) has a degenerate center at $(1,1)$ without loss of generality. Then the HK-system (3) is system (18), $\Delta=0$ and matrix $M$ must be identically zero, that is, the parameters $a, b, c$ and $d$ must satisfy the following equations

$$
\begin{aligned}
& 2 a+3 b+c+4 d=0, \\
& 2 a-b+3 c-4 d=0, \\
& 2 a+b+c-4 d=0 .
\end{aligned}
$$

The solutions of (19) are

$$
b=c=-4 d, \quad a=6 d .
$$

In the following we claim that the equilibrium $(1,1)$ is not a degenerate center of system (18) if $b=c=-4 d$ and $a=6 d$.

When $b=c=-4 d$ and $a=6 d$, system (18) becomes

$$
\begin{aligned}
& \dot{x}=-d x\left(6-4 x+x^{2}-8 y+2 x y+3 y^{2}\right), \\
& \dot{y}=d y\left(6-8 x+3 x^{2}-4 y+2 x y+y^{2}\right) .
\end{aligned}
$$

Of course, $d \neq 0$, otherwise, this system has no isolated equilibria. 
Moving equilibrium $(1,1)$ of system $(20)$ to the origin of the coordinates, we obtain

$$
\begin{aligned}
& \dot{x}=-d(1+x)\left(x^{2}+2 x y+3 y^{2}\right), \\
& \dot{y}=d(1+y)\left(3 x^{2}+2 x y+y^{2}\right) .
\end{aligned}
$$

Doing blow ups of the origin of system (21), let $x=r \cos \theta, y=r \sin \theta$, it can be seen that the local phase portrait of the origin is formed by two hyperbolic sectors, which implies that the origin of system $(21)$ is not center. So equilibrium $(1,1)$ of system (20) is not center. For more information on the changes of variables called blow ups for studying the local phase portraits of the equilibrium points of a planar analytic differential system see for instance section 3.1 of [10].

Therefore, we obtain that HK-system (3) does not have a degenerate center.

For ending the proof of statement (ii) of Theorem 2, it only remains to prove that there do not exist values of the parameters $a, b, c, d, e$ and $f$ such that HK-system (3) has two nilpotent centers.

It is well-known that if an equilibrium is a nilpotent center, then the two eigenvalues of this equilibrium are zero, but the Jacobi matrix at this equilibrium is not identically zero.

Assume that $(1,1)$ is a nilpotent center of HK-system (3), then eigenvalues of the Jacobi matrix $M$ at equilibrium $(1,1)$ are

$$
\pm \sqrt{\frac{1}{2}\left(5 b^{2}+2 c b+12 d b-c^{2}+12 c d+2 a(3 b-c+8 d)\right)},
$$

which must be zero, but $M \neq 0$. We separate the rest of the proof into two cases.

Case I: $3 b-c+8 d=0$.

Case II: $3 b-c+8 d \neq 0$.

Now we consider Case I. Then $c=3 b+8 d$, which leads to the eigenvalues being $\pm|b+4 d| i$. Therefore $b=-4 d$. Plugging the expressions of $c$ and $b$ into $M$, the matrix $M$ becomes

$$
\left(\begin{array}{cc}
a-6 d & a-6 d \\
-a+6 d & -a+6 d
\end{array}\right)
$$

Since $M \neq 0, a-6 d \neq 0$.

Now system (18) becomes

$$
\begin{aligned}
& \dot{x}=-a x+4 d x^{2}+8 d x y-d x^{3}+(a-8 d) x^{2} y-3 d x y^{2}, \\
& \dot{y}=a y-8 d x y-4 d y^{2}+3 d x^{2} y-(a-8 d) x y^{2}+d y^{3} .
\end{aligned}
$$

If $a-12 d \neq 0$, then system (23) has only two equilibria outside the coordinates axes, one is $(1,1)$ and the other is $P=(-a /(a-12 d),-a /(a-12 d))$.

The determinant of Jacobi matrix $M_{p}$ of system (23) at equilibrium $P$ is denoted by $\Delta_{p}$,

$$
\Delta_{p}=-\frac{16 a^{2}(a-6 d)^{2} d}{(a-12 d)^{3}}
$$


We distinguish three subcases: (1.i) $a d \neq 0$, (1.ii) $a=0$ and $d \neq 0$, (1.iii) $d=0$ and $a \neq 0$, to discuss $\Delta_{p}$.

(1.i) If $a d \neq 0$, then $\Delta_{p} \neq 0$, which implies that equilibrium $P$ is either a linear type center or a saddle depending on $\Delta_{p}>0$ or $\Delta_{p}<0$, respectively, i.e. $P$ is not a nilpotent center. Hence, system (23) cannot have two nilpotent centers as $c=3 b+8 d, a-12 d \neq 0$ and $a d \neq 0$.

(1.ii) If $a=0$ and $d \neq 0$, then $\Delta_{p}=0$ and $M_{p}=0$. This leads that $P$ is a degenerated equilibrium. We have proved that the degenerated equilibrium of HK-system (3) cannot be a center, hence, system (23) cannot have two nilpotent centers as $c=3 b+8 d, a=0$ and $d \neq 0$.

(1.iii) If $d=0$ and $a \neq 0$, then $\Delta_{p}=0$ and system (23) becomes

$$
\begin{aligned}
& \dot{x}=-a x(1-x y), \\
& \dot{y}=a y(1-x y),
\end{aligned}
$$

which has non-isolated equilibria. Statement (i) (see C4 in Table 2) has shown that this system has no center. Therefore, system (23) cannot have two nilpotent centers if $c=3 b+8 d$ and $a-12 d \neq 0$.

If $a-12 d=0$, then system (23) has a unique equilibrium at $(1,1)$ outside the coordinates axes. Hence, system (23) cannot have two nilpotent centers as $c=3 b+8 d$ and $a-12 d=0$.

In summary, in Case I we prove that HK-system (3) has a nilpotent equilibrium at $(1,1)$, then this system does not have another nilpotent center which is different from $(1,1)$. Hence HK-system (3) cannot have two nilpotent centers if $3 b-c+8 d=$ 0 .

We now discuss if HK-system (3) has two nilpotent equilibria in Case II: $3 b-$ $c+8 d \neq 0$.

Since $3 b-c+8 d \neq 0$ and the eigenvalues in (22) must be zero in order to have that equilibrium $(1,1)$ is a nilpotent center, we obtain that

$$
a=\frac{c^{2}-5 b^{2}-2 b c-12 b d-12 c d}{2(3 b-c+8 d)} .
$$

Then the Jacobi matrix of system (18) at the equilibrium $(1,1)$ is

$$
\left(\begin{array}{cc}
\frac{(b+4 d)(2 b-c+4 d)}{3 b-c+8 d} & -\frac{(2 b-c+4 d)^{2}}{3 b-c+8 d} \\
\frac{(b+4 d)^{2}}{3 b-c+8 d} & -\frac{(b+4 d)(2 b-c+4 d)}{3 b-c+8 d}
\end{array}\right)
$$

and this equilibrium is nilpotent if and only if $(b+4 d)^{2}+(2 b-c+4 d)^{2} \neq 0$.

We divide three subcases: (2.i) $b+4 d=0$ and $2 b-c+4 d \neq 0$, (2.ii) $b+4 d \neq 0$ and $2 b-c+4 d=0,(2$.iii) $(b+4 d)(2 b-c+4 d) \neq 0$, to study if equilibrium $(1,1)$ is a nilpotent center. 
(2.i) If $b+4 d=0$ and $2 b-c+4 d \neq 0$, then $b=-4 d$. By moving equilibrium $(1,1)$ to the origin, system (18) becomes

$$
\begin{aligned}
\dot{x}= & (c+4 d) y-d x^{2}+(c+2 d) x y+\frac{3}{2}(c+2 d) y^{2}-d x^{3} \\
& -2 d x^{2} y+\frac{3}{2}(c+2 d) x y^{2}, \\
\dot{y}= & 3 d x^{2}+2 d x y-\frac{1}{2}(c+2 d) y^{2}+3 d x^{2} y+2 d x y^{2}-\frac{1}{2}(c+2 d) y^{3} .
\end{aligned}
$$

We must assume that $c+4 d \neq 0$, otherwise the equilibrium $(0,0)$ of system $(24)$ is not nilpotent. Rescaling the independent variable by $c+4 d$ we have system $(24)$ in the normal form for applying Theorem 8 .

$$
\begin{aligned}
\dot{x}= & y-\frac{d}{c+4 d} x^{2}+\frac{c+2 d}{c+4 d} x y+\frac{3(c+2 d)}{2(c+4 d)} y^{2}-\frac{d}{c+4 d} x^{3} \\
& -\frac{2 d}{c+4 d} x^{2} y+\frac{3(c+2 d)}{2(c+4 d)} x y^{2}, \\
\dot{y}= & \frac{3 d}{c+4 d} x^{2}+\frac{2 d}{c+4 d} x y-\frac{c+2 d}{2(c+4 d)} y^{2}+\frac{3 d}{c+4 d} x^{2} y+\frac{2 d}{c+4 d} x y^{2}-\frac{c+2 d}{2(c+4 d)} y^{3} .
\end{aligned}
$$

Using the notation of Theorem 8 we have that

$$
\begin{aligned}
& G(x) \equiv 0, \\
& F(x)=\frac{3 d}{c+4 d} x^{2}+\frac{2 d^{2}}{(c+4 d)^{2}} x^{3}+O\left(x^{4}\right) .
\end{aligned}
$$

Therefore, we must take $d=0$ if we want that the equilibrium $(0,0)$ is a nilpotent center. However, when $d=0$, system (24) becomes

$$
\begin{aligned}
& \dot{x}=\frac{1}{2} c x(y-1)(-1+3 y), \\
& \dot{y}=-\frac{1}{2} c y(y-1)^{2},
\end{aligned}
$$

which has non-isolated equilibria in the line $y=1$ and the $x$-axis is invariant for the flow of this system. Hence, the origin $(0,0)$ cannot become center of system (26), which implies that equilibrium $(1,1)$ is not nilpotent center of system (18) as $b+4 d=0$ and $2 b-c+4 d \neq 0$.

(2.ii) if $b+4 d \neq 0$ and $2 b-c+4 d=0$, Then $c=2 b+4 d$.

Let $x=Y+1, y=X+1$. Then system (18) becomes

$$
\begin{aligned}
\dot{X}= & (b+4 d) Y+3 d Y^{2}+2 d X Y-\frac{1}{2}(b+2 d) X^{2}+3 d X Y^{2} \\
& -(b+2 d) X^{2} Y-\frac{1}{2}(b+2 d) X^{3}, \\
\dot{Y}= & -d Y^{2}+(b+2 d) X Y+\frac{3}{2}(b+2 d) X^{2}-d Y^{3} \\
& +(b+2 d) X Y^{2}+\frac{3}{2}(b+2 d) X^{2} Y .
\end{aligned}
$$

Since $b+4 d \neq 0$, we have system $(27)$ in the normal form for applying Theorem 8 by rescaling the independent variable $b+4 d$. Again using the notation of that theorem we have that $G(X) \equiv 0$ and

$$
F(X)=\frac{3(b+2 d)}{2(b+4 d)} X^{2}+\frac{(b+2 d)^{2}}{2(b+4 d)^{2}} X^{3}+O\left(X^{4}\right) .
$$


Therefore we must take $b=-2 d$ if the origin of system (27) is a nilpotent center. However, when $b=-2 d$, system (27) becomes

$$
\begin{aligned}
& \dot{X}=d Y(1+X)(2+3 Y), \\
& \dot{Y}=-d Y^{2}(1+Y),
\end{aligned}
$$

which has non-isolated equilibria which fill up the $X$-axis. Thus, equilibrium $(0,0)$ cannot be a center of system (28), which leads that equilibrium $(1,1)$ of system (18) is not nilpotent center as $b+4 d \neq 0$ and $2 b-c+4 d=0$.

(2.iii) If $(b+4 d)(2 b-c+4 d) \neq 0$, we shall apply Theorem 8 for proving that the nilpotent equilibrium $(1,1)$ is not a center.

For applying this theorem, we first need to translate the equilibrium $(1,1)$ of system (18) to the origin of coordinates, for this we do the change of variables $x=X+1$ and $y=Y+1$ in system (18), then we shall write the matrix of the linear part at the origin of this system into its real Jordan normal form doing the change of variables

$$
X=-\frac{(c-2 b-4 d)^{2} u}{3 b-c+8 d}-\frac{(2 b-c+4 d) v}{b+4 d}, \quad Y=-\frac{(b+4 d)(2 b-c+4 d) u}{3 b-c+8 d}
$$

Last HK-system $(3)$ in the variables $(u, v)$ can be written as follows

$$
\begin{aligned}
& \dot{u}=v+a_{20} u^{2}+a_{11} u v+a_{02} v^{2}+a_{30} u^{3}+a_{21} u^{2} v+a_{12} u v^{2}, \\
& \dot{v}=b_{20} u^{2}+b_{11} u v+b_{02} v^{2}+b_{30} u^{3}+b_{21} u^{2} v+b_{12} u v^{2}+b_{03} v^{3},
\end{aligned}
$$

where

$$
\begin{aligned}
& a_{20}=\frac{(2 b-c+4 d)\left(b^{2}+2(b+c) d\right)\left(17 b^{2}-14 c b+80 d b+3 c^{2}+96 d^{2}-32 c d\right)}{2(-3 b+c-8 d)^{2}(b+4 d)}, \\
& a_{11}=\frac{2(2 b-c+4 d)\left(2 b^{3}-(c+d) b^{2}+8(c-5 d) d b+d\left(-3 c^{2}+24 d c-64 d^{2}\right)\right)}{(b+4 d)^{2}(3 b-c+8 d)}, \\
& a_{02}=\frac{3 d(2 b-c+4 d)(-3 b+c-8 d)}{(b+4 d)^{3}}, \\
& a_{30}=-\frac{(-2 b+c-4 d)^{2}\left(b^{2}+2(b+c) d\right)\left(17 b^{2}-14 c b+80 d b+3 c^{2}+96 d^{2}-32 c d\right)}{2(3 b-c+8 d)^{3}}, \\
& a_{21}=-\frac{(-2 b+c-4 d)^{2}\left(5 b^{3}-2(c-5 d) b^{2}+16(c-2 d) d b-2 d\left(3 c^{2}-24 d c+32 d^{2}\right)\right)}{(-3 b+c-8 d)^{2}(b+4 d)}, \\
& a_{12}=\frac{3 d(-2 b+c-4 d)^{2}}{(b+4 d)^{2}},
\end{aligned}
$$




$$
\begin{aligned}
& b_{20}=-\frac{3(2 b-c+4 d)\left(b^{2}+2(b+c) d\right)\left(5 b^{2}-4(c-6 d) b+c^{2}+32 d^{2}-8 c d\right)}{2(-3 b+c-8 d)^{2}}, \\
& b_{11}=-\frac{(2 b-c+4 d)\left(b^{2}+2(b+c) d\right)\left(17 b^{2}-14 c b+80 d b+3 c^{2}+96 d^{2}-32 c d\right)}{(-3 b+c-8 d)^{2}(b+4 d)}, \\
& b_{02}=-\frac{(2 b-c+4 d)\left(2 b^{3}-(c+d) b^{2}+8(c-5 d) d b+d\left(-3 c^{2}+24 d c-64 d^{2}\right)\right)}{(b+4 d)^{2}(3 b-c+8 d)}, \\
& b_{30}=\frac{2(b+4 d)(2 b-c+4 d)^{3}\left(b^{2}+2(b+c) d\right)\left(7 b^{2}-5 c b+36 d b+c^{2}+48 d^{2}-12 c d\right)}{(-3 b+c-8 d)^{4}}, \\
& b_{21}=\frac{3(-2 b+c-4 d)^{2}\left(b^{2}+2(b+c) d\right)\left(17 b^{2}-14 c b+80 d b+3 c^{2}+96 d^{2}-32 c d\right)}{2(3 b-c+8 d)^{3}}, \\
& b_{12}=\frac{(-2 b+c-4 d)^{2}\left(5 b^{3}-2(c-5 d) b^{2}+16(c-2 d) d b-2 d\left(3 c^{2}-24 d c+32 d^{2}\right)\right)}{(-3 b+c-8 d)^{2}(b+4 d)}, \\
& b_{03}=-\frac{(-2 b+c-4 d)^{2} d}{(b+4 d)^{2}} .
\end{aligned}
$$

Using the notations introduced in the statement of Lemma 7, we have that

$$
\begin{aligned}
v & =f(u)=-a_{20} u^{2}+\left(a_{11} a_{20}-a_{30}\right) u^{3}+O\left(u^{4}\right), \\
G(u) & \equiv 0, \\
F(u) & =b_{20} u^{2}+\left(b_{30}-a_{20} b_{11}\right) u^{3}+O\left(u^{4}\right) .
\end{aligned}
$$

In order that the equilibrium $(0,0)$ of system $(29)$ is a nilpotent center we must ask

$$
b_{20}=-\frac{3(2 b-c+4 d)\left(b^{2}+2(b+c) d\right)\left(5 b^{2}-4(c-6 d) b+c^{2}+32 d^{2}-8 c d\right)}{2(-3 b+c-8 d)^{2}}=0 .
$$

Since $2 b-c+4 d \neq 0$, we must have

$$
\left(b^{2}+2(b+c) d\right)\left(5 b^{2}-4(c-6 d) b+c^{2}+32 d^{2}-8 c d\right)=0 .
$$

Then either $b^{2}+2(b+c) d=0$ or $5 b^{2}-4(c-6 d) b+c^{2}+32 d^{2}-8 c d=0$.

We now divide three subcases: (II.a) $b^{2}+2(b+c) d=0$ and $b+c \neq 0$, (II.b) $b^{2}+2(b+c) d=0$ and $b+c=0$, (II.c) $b^{2}+2(b+c) d \neq 0$ and $5 b^{2}-4(c-6 d) b+$ $c^{2}+32 d^{2}-8 c d=0$ to discuss if the equilibrium $(1,1)$ of system $(18)$ is a nilpotent center.

Suppose that (II.a) $b^{2}+2(b+c) d=0$ and $b+c \neq 0$. Then $d=-b^{2} /(2(b+c))$.

Pluging the expressions of $a$ and $d$ into system (18), system (18) becomes

$$
\begin{aligned}
& \dot{x}=\frac{x(b x+c y-b-c)(b x+3 c y-b-c)}{2(b+c)}, \\
& \dot{y}=-\frac{y(b x+c y-b-c)(3 b x+c y-b-c)}{2(b+c)},
\end{aligned}
$$

which implies that equilibrium $(1,1)$ is non-isolated since the straight line $b x+c y-$ $b-c=0$ is filled with equilibria, consequently it cannot be a nilpotent center.

Assume that (II.b) $b^{2}+2(b+c) d=0$ and $b+c=0$. Then $b=c=0$ and system (18) becomes

$$
\begin{aligned}
& \dot{x}=-d x(x-3 y)(x-y), \\
& \dot{y}=-d y(-3 x+y)(x-y),
\end{aligned}
$$


which again has a non-isolated equilibrium $(1,1)$ on a straight line $x-y=0$ filled with equilibria.

Now we consider the case (II.c) $b^{2}+2(b+c) d \neq 0$ and $5 b^{2}-4(c-6 d) b+c^{2}+$ $32 d^{2}-8 c d=0$, or equivalently $d=\left(-3 b+c \pm \sqrt{-(b-c)^{2}}\right) / 8$.

Since $d$ is real, we must have $b=c$ and $d=(-3 b+c) / 8$, which implies $3 b-c+8 d=$ 0 . This is not possible because we are in Case $2: 3 b-c+8 d \neq 0$.

In summary, in Case II we prove that HK-system (3) cannot have any a nilpotent equilibrium. Hence HK-system (3) cannot have two nilpotent centers. This completes the proof of statement (ii) of Theorem 2 .

From the proof of nonexistence of two nilpotent centers, we can see that HKsystem (3) can have a nilpotent center. And HK-system (3) has at most two isolated equilibria outside the coordinates axes if there is a nilpotent center. Therefore when HK-system (3) has three or four centers, all of them must be linear type centers. Moreover, if HK-system (3) has only two isolated equilibria outside the coordinates axes, both of them are centers: one is nilpotent and the other is linear type, then they are either in the same quadrant or in the opposite quadrant. Hence, two centers and one of them is nilpotent cannot be in consecutive quadrants.

In the proofs of the rest of statements (iii)-(vi) of Theorem 2 we will give examples showing the existence of HK-systems with centers realizing all possible configurations with respect to the coordinates axes.

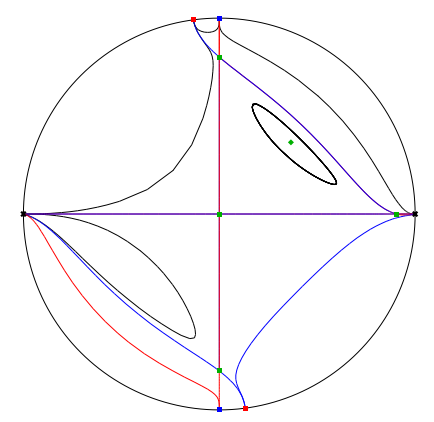

(a) HK-system (30) with a linear type center.

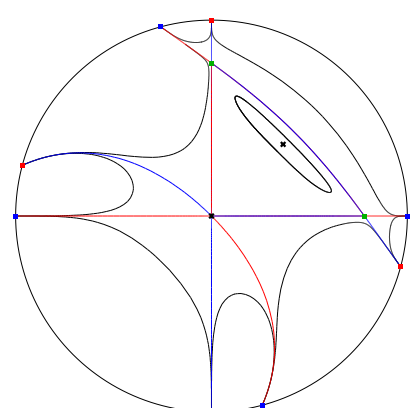

(b) HK-system (31) with a nilpotent center.

Figure 1: HK-systems with a unique center.

Proof of statements (iii) of Theorem 2. The eigenvalues of the linear part of HKsystem (3) at the equilibrium $(1,1)$ are $\pm \sqrt{\Delta / 2}$ where

$$
\Delta=6 a b+5 b^{2}-2 a c+2 b c-c^{2}+16 a d+12 b d+12 c d .
$$

So if $\Delta<0$ the equilibrium $(1,1)$ is a linear type center, because the HK-system is a Hamiltonian system and it cannot have a focus at the equilibrium $(1,1)$. Hence we have proved that there are HK-systems having at least one linear type center. 
Now we shall show that there is a HK-system with a unique center of linear type. Consider the HK-system defined by the Hamiltonian

$$
H=x y\left(-40+4 x+15 x y+2 y^{2}\right) .
$$

The equilibria of this HK-system are

$$
(0,0), \quad(0, \pm 2 \sqrt{5}), \quad(10,0), \quad(1,1) .
$$

So the unique of these equilibria which can be a center is the equilibrium $(1,1)$, which is a linear type center because the eigenvalues of its linear part are $\pm \sqrt{11 / 40} i$. See its global phase portrait in the Poincaré disc in Figure 1(a). For a definition of the Poincaré disc see for instance Chapter 5 of [10].

The real algebraic curve

$$
x y\left(-40+4 x+15 x y+2 y^{2}\right)=h
$$

is a family of level ovals in $\mathbb{R}^{2}$ if and only if $-19<h<0$.

In the next we provide a HK-system with a unique nilpotent center. Consider the HK-system defined by the Hamiltonian

$$
H=x y\left(x+y-\frac{x^{2}}{4}-x y-\frac{y^{2}}{4}\right),
$$

i.e. the HK-system is

$$
\begin{aligned}
& \dot{x}=-x\left(x+2 y-\frac{x^{2}}{4}-2 x y-\frac{3 y^{2}}{4}\right), \\
& \dot{y}=y\left(2 x+y-\frac{3 x^{2}}{4}-2 x y-\frac{y^{2}}{4}\right) .
\end{aligned}
$$

The equilibria of this HK-system are

$$
(0,0), \quad(4,0), \quad(0,4), \quad(1,1) .
$$

Therefore again the unique of these equilibria which can be a center is the equilibrium $(1,1)$. The two eigenvalues of this equilibrium are zero, and since its linear part is not identically zero it is a nilpotent equilibrium. We shall use Theorem 8 for proving that it is a nilpotent center.

First doing the translation $x=X+1$ and $y=Y+1$ we translate the equilibrium $(1,1)$ to the origin of coordinates, and we get the differential system

$$
\begin{aligned}
& \dot{X}=\frac{1}{4}\left(6 X+6 Y+7 X^{2}+14 X Y+3 Y^{2}+X^{3}+8 X^{2} Y+3 X Y^{2}\right), \\
& \dot{Y}=-\frac{1}{4}\left(6 X+6 Y+3 X^{2}+14 X Y+7 Y^{2}+3 X^{2} Y+8 X Y^{2}+Y^{3}\right) .
\end{aligned}
$$

In order to apply Theorem 8 we shall write the linear part at the origin of this system into the real Jordan normal form. So we do the change of variables $X=3 u / 2$ and $Y=v-3 u / 2$, then in the new variables $u$ and $v$ the differential system (33) becomes

$$
\begin{aligned}
& \dot{u}=v-\frac{3}{2} u^{2}+2 u v+\frac{v^{2}}{2}-\frac{9}{4} u^{3}+\frac{3}{4} u^{2} v+\frac{3}{4} u v^{2}, \\
& \dot{v}=-v^{2}+3 u v-\frac{27}{4} u^{3}+\frac{27}{4} u^{2} v-\frac{3}{4} u v^{2}-\frac{1}{4} v^{3} .
\end{aligned}
$$


Now we can apply Theorem 8 to system (34), and using the notation of that theorem we have that $v=f(u)=3 u^{2} / 2+O\left(u^{3}\right)$, and $F(u)=-9 u^{3} / 4+O\left(u^{4}\right)$. Then, since $G(u)=0, a=-9 / 3<0$ and $m=3$, the origin of system (34) is a center or a focus. But since this system has a first integral defined at the origin, the origin is a center. Consequently the equilibrium $(1,1)$ of system (32) is a nilpotent center. See its global phase portrait in the Poincaré disc in Figure 1(b).

The real algebraic curve

$$
x y\left(x+y-\frac{x^{2}}{4}-x y-\frac{y^{2}}{4}\right)=h
$$

is a family of level ovals in $\mathbb{R}^{2}$ if and only if $0<h<\frac{1}{2}$.

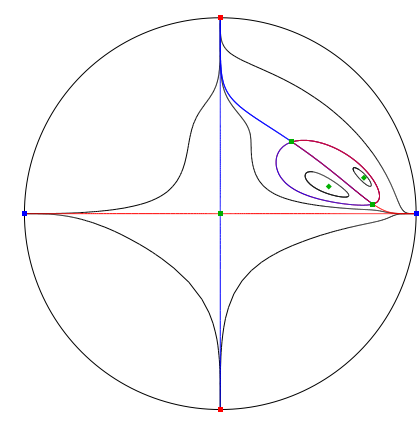

(a) HK-system (35) with two linear type centers.

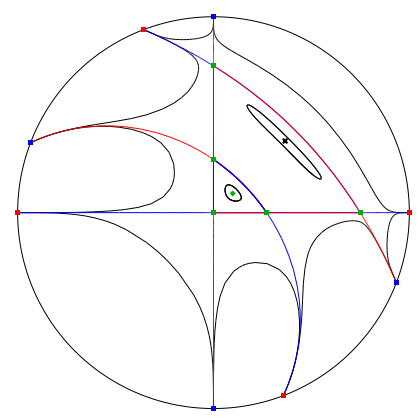

(b) HK-system (36) with one linear type center and one nilpotent center.

Figure 2: HK-systems with two centers in the same quadrant.

Proof of statement (iv) of Theorem 2. First we prove the existence of a HK-system having two linear type centers in the positive quadrant, and no other centers. Consider the HK-system defined by the Hamiltonian

$$
H=x y\left(200-60 x-240 y+6 x^{2}+23 x y+96 y^{2}\right) .
$$

The corresponding HK-system has four equilibria outside the axes of coordinates, namely

$$
p_{1}=(1,1), \quad p_{2}=\left(4, \frac{1}{4}\right), \quad p_{3,4}=\left(\frac{20(9 \pm \sqrt{10})}{71}, \frac{5(9 \pm \sqrt{10})}{71}\right) .
$$

The equilibria $p_{1}$ and $p_{2}$ are hyperbolic saddles because the determinant of their linear parts in both is -1350 , and the equilibria $p_{3,4}$ are linear type centers because the determinant of their linear parts is

$$
\frac{120000(7460 \pm 1531 \sqrt{10})}{357911},
$$


and the system is Hamiltonian. The phase portrait of the HK-system defined by the Hamiltonian (35) is given in Figure 2(a). The real algebraic curve

$$
x y\left(200-60 x-240 y+6 x^{2}+23 x y+96 y^{2}\right)=h
$$

is a family of level ovals in $\mathbb{R}^{2}$ if and only if either $\frac{10000(467-240 \sqrt{10})}{357911}<h<25$ or $25<h<\frac{10000(467+240 \sqrt{10})}{357911}$. Taken together, this real algebraic curve has two families of level ovals in $\mathbb{R}^{2}$ for all real $h$.

Now we provide a HK-system having one linear type center and one nilpotent center in the positive quadrant, and no other centers. The HK-system defined by the Hamiltonian

$$
H=x y\left(2-4 x+x^{2}-4 y+3 x y+y^{2}\right),
$$

has only the following two equilibria outside the axes of coordinates

$$
p_{1}=(1,1) \quad p_{2}=\left(\frac{1}{5}, \frac{1}{5}\right)
$$

The equilibrium $p_{2}$ has eigenvalues $\pm \sqrt{128 / 25} i$, so it is a linear type center.

We shall show that the equilibrium $p_{1}$ is a nilpotent center. The two eigenvalues of this equilibrium are zero, and since its linear part is not identically zero it is a nilpotent equilibrium. In order to study its local phase portrait we translate the equilibrium $p_{1}$ to the origin of coordinates doing the translation $x=X+1$ and $y=Y+1$, and we obtain the differential system

$$
\begin{aligned}
& \dot{X}=4 X-4 Y-5 X^{2}-10 X Y-3 Y^{2}-X^{3}-6 X^{2} Y-3 X Y^{2}, \\
& \dot{Y}=4 X+4 Y+3 X^{2}+10 X Y+5 Y^{2}+3 X^{2} Y+6 X Y^{2}+Y^{3} .
\end{aligned}
$$

Now we shall write the linear part at the origin of this system into its real Jordan normal form, for this we do the change of variables $X=-4 u$ and $Y=4 u+v$. In the new variables $u$ and $v$ the differential system (37) writes

$$
\begin{aligned}
& \dot{u}=v-8 u^{2}-4 u v+\frac{3}{4} v^{2}+32 u^{3}-3 u v^{2}, \\
& \dot{v}=16 u v+2 v^{2}-256 u^{3}-96 u^{2} v+v^{3} .
\end{aligned}
$$

We can apply Theorem 8 to system (38), and using the notation of that theorem we have that $v=f(u)=8 u^{2}+O\left(u^{3}\right)$, and $F(u)=-128 u^{3}+O\left(u^{4}\right)$. Then, since $G(u) \equiv 0, a=-128<0$ and $m=3$, the origin of system (38) is a center. Consequently the equilibrium $p_{1}$ of the Hamiltonian system defined by (36) is a nilpotent center. See its global phase portrait in the Poincaré disc in Figure 2(b). The real algebraic curve

$$
x y\left(2-4 x+x^{2}-4 y+3 x y+y^{2}\right)=h
$$

is a family of level ovals in $\mathbb{R}^{2}$ if and only if either $-1<h<0$ or $0<h<\frac{3}{125}$. In total, this real algebraic curve has two families of level ovals in $\mathbb{R}^{2}$ for all real $h$.

Now we prove the existence of a HK-system having two linear type centers in different opposite quadrants, and no other centers. Consider the HK-system defined by the Hamiltonian

$$
H=x y\left(96+48 x-12 y-32 x^{2}-41 x y-2 y^{2}\right) .
$$




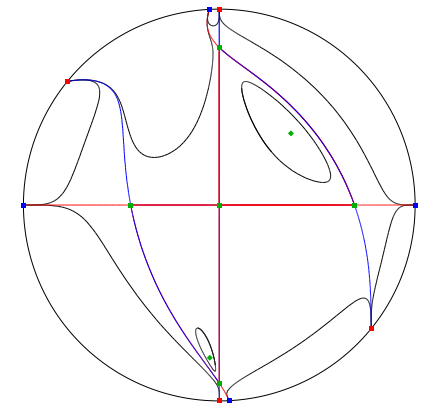

(a) HK-system (39) with two linear type centers.

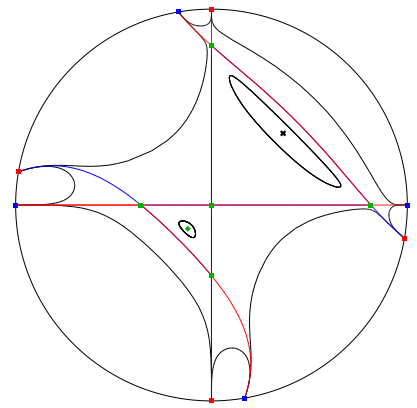

(b) HK-system (40) with one linear type center and one nilpotent center.

Figure 3: HK-systems with two centers in different opposite quadrants.

The corresponding HK-system has two equilibria outside the axes of coordinates, namely

$$
p_{1}=(1,1), \quad p_{2}=\left(-\frac{1}{4},-4\right) .
$$

These two equilibria are linear type centers because the determinant of their linear parts is $475 / 384$ and the system is Hamiltonian. The phase portrait of the HKsystem defined by the Hamiltonian (39) is given in Figure 3(a). The real algebraic curve

$$
x y\left(96+48 x-12 y-32 x^{2}-41 x y-2 y^{2}\right)=h
$$

has two families of level ovals in $\mathbb{R}^{2}$ if and only if $0<h<57$.

Now we exhibit a HK-system having one linear type center and one nilpotent center in different opposite quadrants, and no other centers. Thus the HK-system defined by the Hamiltonian

$$
H=x y\left(4+4 x+4 y-x^{2}-6 x y-y^{2}\right),
$$

has only the following two equilibria outside the axes of coordinates

$$
p_{1}=(1,1) \quad p_{2}=\left(-\frac{1}{4},-\frac{1}{4}\right) .
$$

The linear part of the equilibrium $p_{2}$ has determinant equal to $225 / 64$, so it is a linear type center.

We shall prove that the equilibrium $p_{1}$ is a nilpotent center. Since the two eigenvalues of this equilibrium are zero, and its linear part is not identically zero, it is a nilpotent equilibrium. For studying its local phase portrait we translate the equilibrium $p_{1}$ to the origin of coordinates doing the translation $x=X+1$ and $y=Y+1$, and we obtain the differential system

$$
\begin{aligned}
& \dot{X}=\frac{3}{4}\left(10 X+10 Y+11 X^{2}+22 X Y+3 Y^{2}+X^{3}+12 X^{2} Y+3 X Y^{2}\right), \\
& \dot{Y}=-\frac{3}{4}\left(10 X+10 Y+3 X^{2}+22 X Y+11 Y^{2}+3 X^{2} Y+12 X Y^{2}+Y^{3}\right) .
\end{aligned}
$$


We write the linear part at the origin of this system into its real Jordan normal form doing the change of variables $X=15 u / 2$ and $Y=v-15 u / 2$. In the new variables $u$ and $v$ the differential system (41) writes

$$
\begin{aligned}
& \dot{u}=v+\frac{1}{20}\left(-900 u^{2}+240 u v+6 v^{2}-6750 u^{3}+675 u^{2} v+45 u v^{2}\right), \\
& \dot{v}=-\frac{3}{4}\left(-120 u v+8 v^{2}+6750 u^{3}-1350 u^{2} v+45 u v^{2}+v^{3}\right) .
\end{aligned}
$$

Applying Theorem 8 to system (42), and using the notation of that theorem we have that $v=f(u)=45 u^{2}+O\left(u^{3}\right)$, and $F(u)=-101258 u^{3} / 2+O\left(u^{4}\right)$. Then, since $G(u)=0, a=-10125 / 2<0$ and $m=3$, the origin of system (42) is a center. Consequently the equilibrium $p_{1}$ of the Hamiltonian system defined by (40) is a nilpotent center. See its global phase portrait in the Poincaré disc in Figure 3(b). The real algebraic curve

$$
x y\left(4+4 x+4 y-x^{2}-6 x y-y^{2}\right)=h
$$

has two families of level ovals in $\mathbb{R}^{2}$ if and only if $0<h<\frac{3}{32}$. And when $\frac{3}{32} \leq h<4$, $x y\left(4+4 x+4 y-x^{2}-6 x y-y^{2}\right)=h$ has a family of level ovals in $\mathbb{R}^{2}$.

In summary, for all $h \in \mathbb{R}$ the real algebraic curve $x y\left(4+4 x+4 y-x^{2}-6 x y-y^{2}\right)=$ $h$ has two families of level ovals in $\mathbb{R}^{2}$.

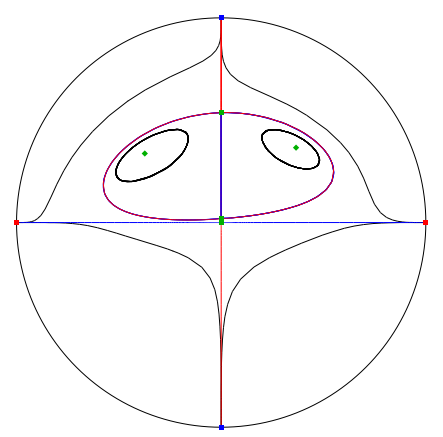

Figure 4: HK-systems with two centers in different consecutive quadrants.

We provide a HK-system having two linear type centers in different consecutive quadrants, and no other centers. Consider the HK-system defined by the Hamiltonian

(43) $H=x y\left(178074+356148 x-4989116 y+558568 x^{2}-404079 x y+3231200 y^{2}\right)$.

The corresponding HK-system has two equilibria outside the axes of coordinates, namely

$$
(1,1), \quad\left(-1, \frac{361}{400}\right)
$$

These two equilibria are linear type centers because the determinants of their linear parts are

$$
6889484999096 \text { and } \frac{461859088701689761}{80000}
$$


respectively. The phase portrait of the HK-system defined by the Hamiltonian (43) is given in Figure 4. This completes the proof of statement (d) of Theorem 2. The real algebraic curve

$$
x y\left(178074+356148 x-4989116 y+558568 x^{2}-404079 x y+3231200 y^{2}\right)=h
$$

has a family of level ovals in $\mathbb{R}^{2}$ if and only if either $-1069205<h<0$ or $0<h<$ $\frac{162546646559}{160000}$.

In summary, for all $h \in \mathbb{R}$ the real algebraic curve $x y(178074+356148 x-$ $\left.4989116 y+558568 x^{2}-404079 x y+3231200 y^{2}\right)=h$ has two families of level ovals in $\mathbb{R}^{2}$.

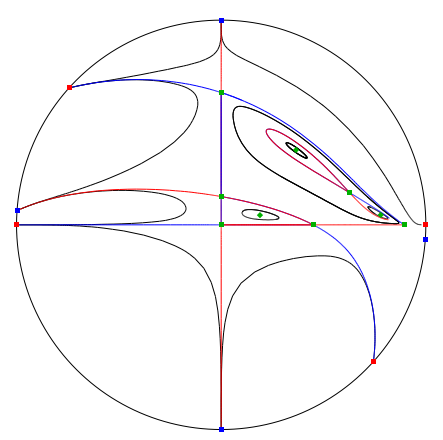

Figure 5: HK-systems with three linear type centers in the same quadrant.

Proof of statement $(v)$ of Theorem 2. We show a HK-system having three linear type centers in the same quadrant, and no other centers. Let the HK-system be defined by the Hamiltonian

$$
H=x y\left(20-20 x-80 y+2 x^{2}+31 x y+32 y^{2}\right) .
$$

The associated HK-system has four equilibria outside the axes of coordinates, namely

$$
(1,1), \quad\left(4, \frac{1}{4}\right), \quad\left(\frac{2}{47}(30 \mp \sqrt{430}), \frac{1}{94}(30 \mp \sqrt{430})\right) .
$$

The determinants of their linear parts are

$$
\frac{27}{10}, \quad \frac{27}{10} \text { and } \frac{4( \pm 995 \sqrt{430}-13588)}{103823},
$$

respectively. Therefore the three first equilibria of (45) are linear type centers and the fourth one is a hyperbolic saddle.

The real algebraic curve

$$
x y\left(20-20 x-80 y+2 x^{2}+31 x y+32 y^{2}\right)=h
$$


has two families of level ovals in $\mathbb{R}^{2}$ if and only if $-15<h<-\frac{100(6889+344 \sqrt{430})}{103823}$, and it has a family of level ovals in $\mathbb{R}^{2}$ if and only if either $-\frac{100(6889+344 \sqrt{430})}{103823} \leq h<0$ or $0<h<\frac{100(-6889+344 \sqrt{430})}{103823}$.

In summary, for all $h \in \mathbb{R}$ the real algebraic curve $x y\left(20-20 x-80 y+2 x^{2}+\right.$ $\left.31 x y+32 y^{2}\right)=h$ has four families of level ovals in $\mathbb{R}^{2}$. In Figure 5 we show the phase portrait of the HK-system defined by the Hamiltonian in (44).

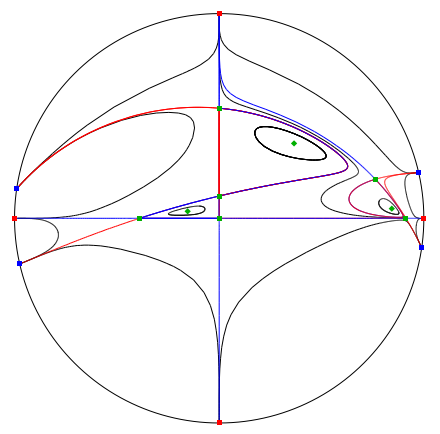

Figure 6: HK-systems with two linear type centers in the same quadrant and the other in a consecutive quadrant.

Now we provide a HK-system having two linear type centers in the same quadrant and the other in a consecutive quadrant, and no other centers. Let the HK-system be defined by the Hamiltonian

$$
H=x y\left(-20-20 x+104 y+2 x^{2}+5 x y-60 y^{2}\right) .
$$

The associated HK-system has four equilibria outside the axes of coordinates, namely

$$
(4,1), \quad(1,1), \quad\left(\frac{2(56071 \mp 829 \sqrt{6157})}{101(485 \pm \sqrt{6157})}, \frac{1}{606}(121 \mp \sqrt{6157})\right) .
$$

The determinants of their linear parts are

$$
-\frac{108}{5}, \quad \frac{63}{10} \text { and } \frac{658(731635 \pm 9034 \sqrt{6157})}{46363545},
$$

respectively. Therefore the first equilibrium of (47) is a hyperbolic saddle and the other three equilibria are linear type centers. In Figure 6 we show the phase portrait of the HK-system defined by the Hamiltonian (46). The phase portrait shows that the real algebraic curve

$$
x y\left(-20-20 x+104 y+2 x^{2}+5 x y-60 y^{2}\right)=h
$$

has three families of level ovals in $\mathbb{R}^{2}$ for all $h \in \mathbb{R}$, in which this real algebraic curve has two families of level ovals if and only if $0<h<\frac{94(-2956253+37859 \sqrt{6157})}{9272709}$, and it has a family of level ovals if and only if either $\frac{94(-2956253+37859 \sqrt{6157})}{9272709} \leq h<11$ or $-\frac{94(2956253+37859 \sqrt{6157})}{9272709}<h<-16$. 


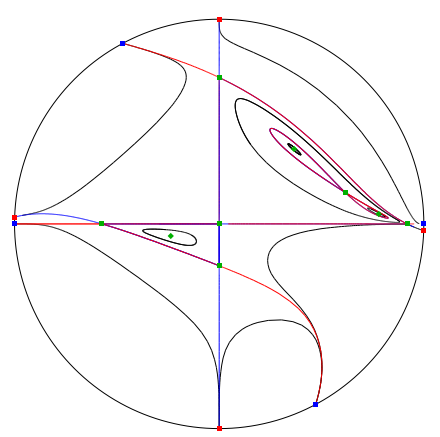

Figure 7: HK-systems with three linear type centers two in the same quadrant and the other in the opposite quadrant.

We give a HK-system having three linear type centers, two in the same quadrant and the other in the opposite quadrant, and no other centers. Consider the HKsystem defined by the Hamiltonian

$$
H=x y\left(-40-20 x-80 y+2 x^{2}+61 x y+32 y^{2}\right) .
$$

The corresponding HK-system has four equilibria outside the axes of coordinates, namely

$$
(1,1), \quad\left(4, \frac{1}{4}\right), \quad\left(\frac{4}{77}(15 \mp \sqrt{610}), \frac{1}{77}(15 \mp \sqrt{610})\right) .
$$

The determinants of their linear parts are

$$
\frac{81}{40}, \quad \frac{81}{40} \text { and } \frac{4( \pm 2995 \sqrt{610}-30988)}{456533},
$$

respectively. Therefore the three first equilibria of (49) are linear type centers and the fourth one is a hyperbolic saddle. The phase portrait of the HK-system defined by the Hamiltonian (48) is given in Figure 7. The phase portrait shows that the real algebraic curve

$$
x y\left(-40-20 x-80 y+2 x^{2}+61 x y+32 y^{2}\right)=h
$$

has four families of level ovals in $\mathbb{R}^{2}$ for all $h \in \mathbb{R}$, in which this real algebraic curve has two families of level ovals if and only if either $-45<h<-\frac{400(25189+976 \sqrt{610})}{456533}$ or $\frac{400(-25189+976 \sqrt{610})}{456533}<h<0$, and it has a family of level ovals if and only if $-\frac{400(25189+976 \sqrt{610})}{456533} \leq h \leq \frac{400(-25189+976 \sqrt{610})}{456533}$.

We show a HK-system having three linear type centers in three different quadrants, and no other centers. Consider the HK-system defined by the Hamiltonian

$$
H=x y\left(-108-108 x+32 y+72 x^{2}+37 x y+2 y^{2}\right) .
$$

The corresponding HK-system has four equilibria outside the axes of coordinates, namely

$$
(1,1), \quad\left(-\frac{1}{4},-9\right), \quad\left(\frac{2}{793}(-606 \pm 275 \sqrt{3}), \frac{27}{793}(239 \mp 115 \sqrt{3})\right) .
$$




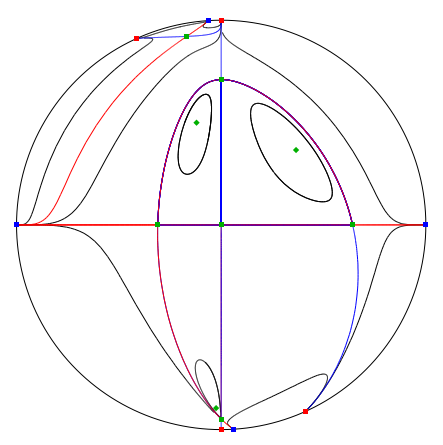

Figure 8: HK-systems with three linear type centers in three different quadrants.

The determinants of their linear parts are

$$
\frac{2425}{288}, \quad \frac{3925}{1458} \text { and }-\frac{47375215450}{498677257} \pm \frac{27501846750 \sqrt{3}}{498677257},
$$

respectively. Therefore the three first equilibria of (51) are linear type centers and the fourth one is a hyperbolic saddle. In Figure 8 we show the phase portrait of the HK-system defined by the Hamiltonian (50). The real algebraic curve

$$
x y\left(-108-108 x+32 y+72 x^{2}+37 x y+2 y^{2}\right)=h
$$

has three families of level ovals in $\mathbb{R}^{2}$ for all $h \in \mathbb{R}$, in which this real algebraic curve has two families of level ovals if and only if $-73<h<0$, and it has a family of level ovals if and only if either $-\frac{4293}{16}<h \leq-73$ or $0<h<\frac{5832(-241463148+140160125 \sqrt{3})}{498677257}$.

This completes the proof of statement $(v)$ of Theorem 2.

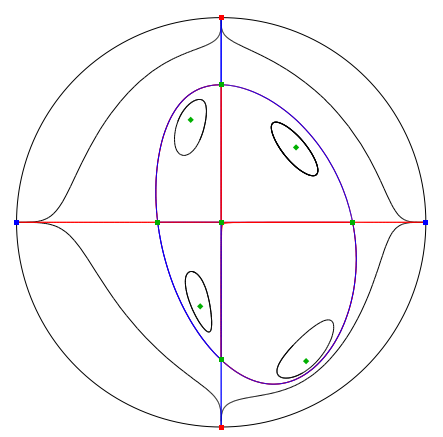

Figure 9: HK-systems with four linear type centers in four different quadrants.

Proof of statement (vi) of Theorem 2. From the proof of the nonexistence of two nilpotent centers, we can see that the four centers must be linear if a HK-system 
(3) has four centers. And we claim that this system has and only has four families of periodic orbits, one of which contains a linear type center in its interior.

In fact, if a HK-system (3) has four centers, then this system has four families of periodic orbits, one of which contains a linear type center in its interior by the definition of center.

On the other hand, if a HK-system (3) has four centers, then all other equilibria of this system should be on the $x$-axis or the $y$-axis, i.e. this system cannot have the other equilibria in the interior of quadrants of $\mathbb{R}^{2}$ by Theorem 6 and statement (i) of Theorem 2. Note that the $x$-axis and $y$-axis are invariant for the flow of the HK-system (3). The periodic orbit of the HK-system (3) cannot intersect with the $x$-axis or the $y$-axis. Hence, there does not exist a periodic orbit of this system such that the periodic orbit contains more than two equilibria in its interior due to the sum of indices of these equilibria being one and the index of a linear type center being one. Therefore a HK-system having four linear type centers has and only has four families of periodic orbits, one of which contains a linear type center in its interior. This leads that the level set of the corresponding Hamiltonian

$$
x y\left(a+b x+c y+d x^{2}+e x y+f y^{2}\right)=h
$$

has four families of level ovals in $\mathbb{R}^{2}$.

We now face an interesting problem what are configurations of four centers with respect to the coordinates axes if a HK-system (3) has four centers. In the following, we provide a HK-system having four linear type centers in four different quadrants. Let the HK-system be defined by the Hamiltonian

$$
H=x y\left(-12-12 x+8 x^{2}+5 x y+2 y^{2}\right) .
$$

The associated HK-system has four equilibria outside the axes of coordinates, namely

$$
(1,1),\left(-\frac{1}{4},-1\right),\left(\frac{2}{13}(6 \pm 5 \sqrt{3}), \frac{1}{13}(-15 \mp 19 \sqrt{3})\right)
$$

one equilibrium is in different quadrant. The determinants of their linear parts are

$$
\frac{23}{6}, \quad \frac{83}{96} \text { and } \frac{2(30191 \pm 16525 \sqrt{3})}{2197},
$$

respectively. All these determinants are positive. Hence the four equilibria of (53) are linear type centers. In Figure 9 there is the phase portrait of the HK-system defined by the Hamiltonian (52). The real algebraic curve

$$
x y\left(-12-12 x+8 x^{2}+5 x y+2 y^{2}\right)=h
$$

has fours families of level ovals in $\mathbb{R}^{2}$ for all $h \in \mathbb{R}$, in which this real algebraic curve has two families of level ovals if and only if either $-\frac{21}{16}<h<0$ or $0<h<$ $\frac{72(1660-911 \sqrt{3})}{2197}$, and it has a family of level ovals if and only if either $-9<h \leq-\frac{21}{16}$ or $\frac{72(1660-911 \sqrt{3})}{2197} \leq h<\frac{72(1660+911 \sqrt{3})}{2197}$.

We show a HK-system having four linear type centers, two in the same quadrant, and each one of the other two centers is located in a different adjacent quadrat. Let the HK-system be defined by the Hamiltonian

$$
H=x y\left(57-456 x+270 y+592 x^{2}-710 x y+229 y^{2}\right) .
$$




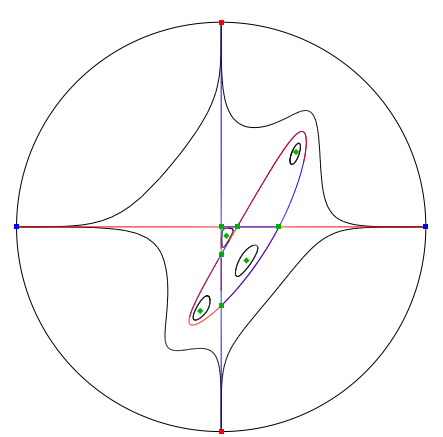

Figure 10: HK-systems with four linear type centers, two in the same quadrant, and each one of the other two centers is located in a different adjacent quadrat.

The associated HK-system has four equilibria outside the axes of coordinates, namely

$$
(1,1),\left(-\frac{1}{4},-1\right),\left(\frac{72732 \mp 5 \sqrt{95939265}}{470788}, \frac{19(-16455 \pm \sqrt{95939265)}}{1456898}\right) .
$$

The determinants of their linear parts are

$$
\frac{5159}{8664}, \quad \frac{367}{4332} \text { and } \frac{5(109357753909491323 \mp 8468817930191 \sqrt{95939265)}}{36973060615773331024},
$$

respectively. All these determinants are positive. Therefore the four equilibria of (55) are linear type centers. In Figure 10 there is the phase portrait of the HK-system defined by the Hamiltonian (54). The real algebraic curve

$$
x y\left(57-456 x+270 y+592 x^{2}-710 x y+229 y^{2}\right)=h
$$

has fours families of level ovals in $\mathbb{R}^{2}$ for all $h \in \mathbb{R}$, in which this real algebraic curve has three families of level ovals if and only if

$$
\frac{16245(2340735907022315-256172312597 \sqrt{95939265})}{36973060615773331024}<h<0,
$$

it has two families of level ovals if and only if

$$
-\frac{21}{8}<h \leq \frac{16245(2340735907022315-256172312597 \sqrt{95939265})}{36973060615773331024},
$$

and it has a family of level ovals if and only if either $-18<h \leq-\frac{21}{8}$ or $0<h<$ $\underline{16245(2340735907022315+256172312597 \sqrt{95939265)}}$.

From our results it remains the following open question.

Open question: Are the two configurations of four centers with respect to the coordinates axes described in statement (vi) of Theorem 2 the unique which are realizable with four centers by the HK-systems? 


\section{ACKnowledgements}

We are grateful to the anonymous referee for the careful reading and valuable comments which led to improvements of our original manuscript.

The first author is partially supported by a FEDER-MINECO grants numbers MTM2016-77278-P and MTM2013-40998-P, an AGAUR grant number 2014SGR568, and the grants FP7-PEOPLE-2012-IRSES 318999, and from the recruitment program of high-end foreign experts of China.

The second author is partially supported by the National Natural Science Foundations of China (No.11371248 \& No. 11431008) and the RFDP of Higher Education of China grant (No.20130073110074).

\section{REFERENCES}

[1] A.F. ANDREEV, Investigation of the behaviour of the integral curves of a system of two differential equations in the neighbourhood of a singular point, Amer. Math. Soc. Transl. (2) 8 (1958), 183-207.

[2] V.I. Arnold, Mathematical Methods of Classical Mechanics, Graduate Texts in Mathematics, Vol. 60, Springer-Verlag, New York, 1997.

[3] N.N. BAUTin, On the number of limit cycles which appear with the variation of coefficients from an equilibrium position of focus or center type, Mat. Sbornik 30 (1952), 181-196, Amer. Math. Soc. Transl. Vol. 100 (1954), 1-19.

[4] C.A. Buzzi, J. Llibre And J.C. Medrado, Phase portraits of reversible linear differential systems with cubic homogeneous polynomial nonlinearities having a non-degenerate center at the Origin, Qual. Theory Dyn. Syst. 7 (2009), 369-403.

[5] I.E. Colak, J. Llibre AND C. Valls, Hamiltonian linear type centers of linear plus cubic homogeneous polynomial vector fields, J. Differential Equations 257 (2014), 1623-1661.

[6] I.E. Colak, J. Llibre AND C. VAlls, Hamiltonian nilpotent centers of linear plus cubic homogeneous polynomial vector fields, Advances in Mathematics 259 (2014), 655-687.

[7] I.E. Colak, J. Llibre AND C. VAlls, Bifurcation diagrams for Hamiltonian linear type centers of linear plus cubic homogeneous polynomial vector fields, J. of Differential Equations 258 (2015), 846-879.

[8] I.E. Colak, J. Llibre and C. VAlls, Bifurcation diagrams for Hamiltonian nilpotent centers of linear plus cubic homogeneous polynomial vector fields, J. Differential Equations 262 (2017), 5518-5533.

[9] H. DulaC, Détermination et integration d'une certaine classe d'équations différentielle ayant par point singulier un centre, Bull. Sci. Math. Sér. (2) 32 (1908), 230-252.

[10] F. Dumortier, J. Llibre And J.C. Artés, Qualitative theory of planar differential systems, Universitext, Springer-Verlag, 2006.

[11] W. Fulton, Algebraic Curves, Mathematics Lecture Note Series, W.A. Benjamin, 1974.

[12] D. Hilbert, Mathematische Probleme, Lecture, Second Internat. Congr. Math. (Paris, 1900), Nachr. Ges. Wiss. G"ottingen Math. Phys. KL. (1900), 253-297; English transl., Bull. Amer. Math. Soc. 8 (1902), 437-479; Bull. (New Series) Amer. Math. Soc. 37 (2000), 407-436.

[13] J. Hofbauer And K. Sigmund, The Theory of Evolution and Dynamical Systems, Cambridge Univ. Press, Cambridge, UK, 1988.

[14] Yu. Ilyashenko, Centennial history of Hilbert's 16th problem, Bull. (New Series) Amer. Math. Soc. 39 (2002), 301-354.

[15] W. KAPTEYN, On the midpoints of integral curves of differential equations of the first degree, Nederl. Akad. Wetensch. Verslag. Afd. Natuurk. Konikl. Nederland (1911), 1446-1457 (Dutch).

[16] W. KAPTEYN, New investigations on the midpoints of integrals of differential equations of the first degree, Nederl. Akad. Wetensch. Verslag Afd. Natuurk. 20 (1912), 1354-1365; 21, 27-33 (Dutch).

[17] N.A. Kolmogorov, Sulla teoria di Volterra della lotta per l'esistenza, Giorn. Istituto Ital. Attuari, 7 (1936), 74-80. 
[18] J. LI, Hilbert's 16th problem and bifurcations of planar polynomial vector fields, Internat. J. Bifur. Chaos Appl. Sci. Engrg. 13 (2003), 47-106.

[19] J. Llibre, Centers: their integrability and relations with the divergence, Applied Mathematics and Nonlinear Sciences 1 (2016), 79-86.

[20] A. J. LотkA, Elements of Physical Biology, Williams and Wilkins, Baltimore, MD, 1925.

[21] K.E. Malkin, Criteria for the center for a certain differential equation, (Russian) Volz. Mat. Sb. Vyp. 2 (1964), 87-?91.

[22] H. Poincaré, Mémoire sur les courbes définies par une équation differentielle, J. Maths. Pures Appl. 7 1881, 375-422.

[23] D. Schlomiuk, Algebraic particular integrals, integrability and the problem of the center, Trans. Amer. Math. Soc. 338 (1993), 799-841.

[24] S. Smale, On the differential equations of species in competition, J. Math. Biology, 3 (1976), $5-7$.

[25] V. Volterra, Variazioni e fluttuaziono del numero di individui in specie animali conviventi, Mem. Accad. Lincei 2 (1926), 31-113.

[26] N. I. VulPE, Affine-invariant conditions for the topological discrimination of quadratic systems with a center, Differential Equations 19 (1983), 273-280.

[27] N.I. Vulpe And K.S. SibirskiI, Centro-affine invariant conditions for the existence of a center of a differential system with cubic nonlinearities, (Russian) Dokl. Akad. Nauk SSSR 301 (1988), 1297-1301; translation in Soviet Math. Dokl. 38 (1989), 198-201

[28] Z. Zhang, T. Ding, W. Huang And Z. Dong, Qualitative Theory of Differential Equations, Translations of Mathematical Monographs 101, Amer. Math. Soc., Providence, 1991.

[29] H. ŻOŁA̧DEK, Quadratic systems with center and their perturbations, J. Differential Equations 109 (1994), 223-273.

[30] H. ŻoŁA̧DEK, On a certain generalization of Bautin's theorem, Nonlinearity 7 (1994), 273279 .

[31] H. ŻoŁA̧DEK, The classification of reversible cubic systems with center, Topol. Methods Nonlinear Anal. 4 (1994), 79-136.

[32] H. ŻOŁA̧DEK, Remarks on: "The classification of reversible cubic systems with center, Topol. Methods Nonlinear Anal. 4 (1994), 79-136", Topol. Methods Nonlinear Anal. 8 (1996), 335342 .

1 Departament de Matemátiques, Universitat Autónoma de Barcelona, 08193 Bellaterra, Barcelona, Catalonia, Spain; and School of Mathematical Sciences, Shanghai Jiao Tong University, Shanghai 200240, China

E-mail address: jllibre@mat.uab.cat

2 School of Mathematical Sciences, Shanghai Jiao Tong University, Shanghai 200240 , CHINA

E-mail address: xiaodm@sjtu.edu.cn 